\title{
A Case of Acquired Giant Digital Fibrokeratoma
}

Keywords: Acquired giant digital fibrokeratoma; Great toe

\section{Introduction}

Acquired digital fibrokeratoma is a rare benign tumor which was first named to describe digital lesions not present at birth, and histopathologically consistent with fibrokeratoma. It usually occurs in adults as solitary lesion and most frequent sites are the fingers and toes. Generally, the size of fibrokeratoma is small, usually $3 \sim 5$ $\mathrm{mm}$. Although the standard to define the term 'giant' has not been established, in previous reports, the acquired digital fibrokeratoma larger than $1 \mathrm{~cm}$ have been reported as giant digital fibrokeratomas. Herein, we report a case of giant digital fibrokeratoma occurring on the left great toe.

\section{Case Report}

An otherwise healthy 79-year-old man presented with solitary nodule on his left great toe. It was painful and it affected patient's mobility. The lesion was developed 8 years ago and he said that lesion had been slowly growing. There was no history of trauma. On cutaneous examination a solitary, $2.5 * 2.0 * 1.0 \mathrm{~cm}$-sized, broadbased, firm skin-colored mass was noticed on the lateral side of his left great toe (Figure 1). It was well-demarcated and not pulsatile. $\mathrm{X}$-ray did not show any bony structures inside the lesion. Under local anesthesia, complete excision of the mass was made. Histopathologic examination showed hyperkeratosis and acanthosis in the epidermis. The dermis showed thick collagen bundles with dilated capillaries oriented in the direction of the longitudinal axis (Figure 2). On basis of the clinical and histopathological features, diagnosis of giant digital fibrokeratoma was made. The excision site was completely healed without any recurrence for 1 year.

\section{Discussion}

The acquired digital fibrokeratoma is a rare tumor which was first reported by Bart et al. in 1968 [1]. For its distinct histopathologic features, the authors named this tumor as an acquired digital fibrokeratoma (ADFK). The lesions are usually solitary, dome-shaped, although they may present as elongated finger-like projections. They do not spontaneously resolve and predominantly located on the fingers and toes, but occurring on the lower lip, nose, elbow, prepatellar area, nail bed and heel were reported [2-4]. The size of the tumor is generally small, approximately $3 \sim 5 \mathrm{~mm}$. Although a precise standard size to define a giant ADFK has not been established, ADFK larger than $1 \mathrm{~cm}$ is often regarded as 'giant'. There have been six literatures reporting a giant ADFKs, and their size were $3.2 * 3.8^{*} 1.5$ $\mathrm{cm}, 3.0 * 1.5 \mathrm{~cm}, 1^{\star} 1 * 3 \mathrm{~cm}, 2.5 * 1.6 * 1.4 \mathrm{~cm}, 1^{\star} 1 * 3 \mathrm{~cm}$, and $3.0 *$ $2.2 * 1.0 \mathrm{~cm}$ [5]. They were all located on the foot or toes. In our case, the size of the tumor was $2.5 * 2.0 * 1.0 \mathrm{~cm}$ and located on the left great toe, so we can regard it as giant ADFK.

The pathophysiology of an ADFK is unknown. It is presumed that trauma result in the development of ADFK, especially on digits [6]. Moreover, Nemeth and Penneys reported that Factor XIIIa, which is

\section{Journal of}

\section{Clinical \& Investigative} Dermatology

\section{Seok Hoon Moon, Sang Hyun Cho, Jeong Deuk Lee and Hei Sung Kim*}

Department of Dermatology, Catholic University of Korea, Incheon St. Mary's Hospital, Incheon, Korea

\section{*Address for Correspondence}

Hei Sung Kim, Department of Dermatology, Catholic University of Korea, Incheon St. Mary's Hospital, Incheon, Korea, Tel: 82-32-280-5700; E-mail: hazelkimhoho@gmail.com

Submission: 19 September, 2016

Accepted: 04 October, 2016

Published: 13 October, 2016

Copyright: $\odot 2016 \mathrm{Kim}$ HS, et al. This is an open access article distributed under the Creative Commons Attribution License, which permits unrestricted use, distribution, and reproduction in any medium, provided the original work is properly cited.

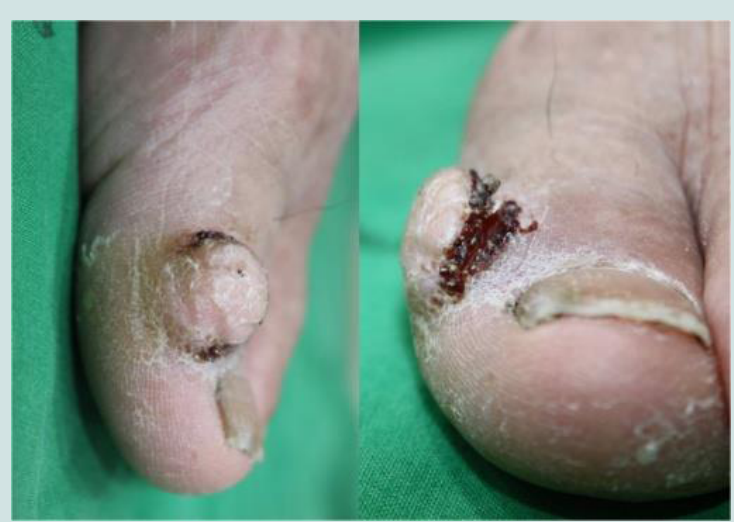

Figure 1: Solitary, 2.5 * 2.0 * $1.0 \mathrm{~cm}$-sized, broad-based, firm skin-colored mass on the lateral side of the left great toe.

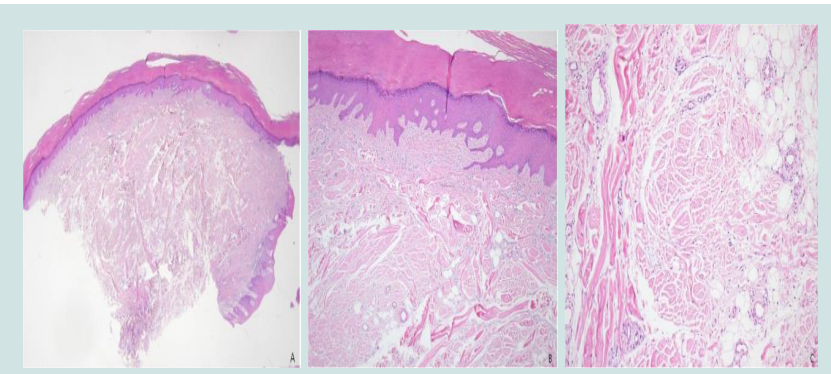

Figure 2: Hyperkeratosis and acanthosis in the epidermis. The dermis showed thick collagen bundles and fibroblasts with dilated capillaries oriented in the direction of the longitudinal axis.

found in fibrovascular tumors, was also found in ADFK, suggesting that this factor might play an important role in developing the tumor [7].

Kint et al. examined 50 cases of ADFK and described 3 types of ADFK by their clinical and histopathologic features [8]. All types of ADFK were characterized by a very hyperkeratotic stratum corneum. 
Type 1 ADFK is a dome-shaped lesion which contains fibroblast between collagen bundles, fine elastic fibers and numerous capillaries in dermis. Type II ADFK is generally tall and hyperkeratotic lesion which contains more fibroblasts and less elastic fibers than type I ADFK. Type III ADFK is a flat to dome-shaped lesion characterized by poorly cellular and edematous structures and no elastic fibers. This case showed the typical histopathologic findings of type III ADFK with poor cellular structure and absent elastic tissue.

The differential diagnoses to consider include cutaneous horn, neurofibroma and supernumerary digits. Although cutaneous horns resemble ADFKs clinically, they do not have a prominent core of outgrowing connective tissue and often have epidermal neoplasia at the base. Neurofibromas present as soft and polypoid skin-colored mass and contains loosely spaced spindle cells and wavy collagenous strands in the dermis. Supernumerary digit is a congenital digital anomaly which usually observed at the base of the $5^{\text {th }}$ digit and contains neural bundles in the dermis [5].

The optimal treatment of an ADFK is complete excision to include the basal attachment. No recurrence is reported after complete surgical excision [9]. The diagnosis of abnormal growth on the toe can be challenging. ADFK should be considered as part of the differential of a hyperkeratotic lesion on one of the digits, particularly of a persistent lesion that does not resolve. Herein, we report a case of giant ADFK occurred on the left great toe as a rare case.

\section{References}

1. Bart RS, Andrade R, Kopf AW, Leider M (1968) Acquired digital fibrokeratomas. Arch Dermatol 97: 120-129.

2. Kim HJ, Lee SH, Park EJ, Kim CW, Jo HJ, et al. (2006) A case of acquired fibrokeratoma of the lower lip. Korean J Dermatol 44: 1438-1440.

3. Hur J, Suh KS, Kim ST (2000) Two cases of acquired fibrokeratoma. Korean J Dermatol 38: 1116-1117.

4. Jee MS, Lee DP, Chang SE, Choi JH, Sung KJ, et al. (2003) Two cases of acquired fibrokeratoma occuring on the prepatellar area and the nail bed. Korean J Dermatol 41: 403-405.

5. Ali M, Mbah CA, Alwadiya A, Nur MM, Sunderamoorthy D (2015) Giant fibrokeratoma, a rare soft tissue tumor presenting like an accessory digit, a case report and review of literature. Int J Surg Case Rep 10: 187-190.

6. Altman DA, Griner JM, Faria DT (1994) Acquired digital fibrokeratoma. Cutis 54: 93-94.

7. Nemeth AJ, Penneys NS (1989) Factor XIIla is expressed by fibroblasts in fibrovascular tumors. J Cutan Pathol 16: 266-271.

8. Kint A, Baran R, De Keyser H (1985) Acquired (digital) fibrokeratoma. J Am Acad Dermatol 12(5 Pt 1): 816-821.

9. Choi JH, Jung SY, Chun JS, Seo JK, Lee D, et al. (2011) Giant acquired digital fibrokeratoma occurring on the left great toe. Ann Dermatol 23: 64-66.

\section{Acknowledgements}

Funding Sources: This study was supported by a grant of the Korean Healthcare technology R\&D project, Ministry of Health \& Welfare, Republic of Korea (Grant No.: NH15C0105). 